\title{
Le genre Nippostrongylus Lane, 1923, (Nématode - Héligmosomatidé)
}

\author{
par Marie-Claude DURETTE-DESSET
}

Laboratoire de Zoologie (Vers), associé au C.N.R.S. (Pr A.-G. ChaвaUd), Muséum National d'Histoire Naturelle, 57, rue Cuvier, F. 75, Paris, $5^{\text {e }}$

\section{Résumé}

Le genre Nippostrongylus Lane, 1923 est redéfini en tenant compte du synlophe. Il est caractérisé essentiellement par l'orientation et le gradient des arêtes cuticulaires ainsi que la forte asymétrie de la bourse caudale.

Nous y regroupons six espèces qui, excepté $N$. rauschi (Chabaud et Desset, 1966), parasite de Dermoptères, sont inféodées à certains Muridés orientaux et australiens.

\section{Summary}

The genus Nippostrongylus Lane, 1923 (Nematoda, Heligmosomatidae).

A redefinition of the genus Nippostrongylus Lane, 1923 has been worked out on the basis of its synlophe morphology. It is readily characterised by its cuticular ridges arrangement and size gradient, and its strongly asymetrical caudal bursa.

Six species are referred to this genus, with the exception of $N$. rauschi (Chabaud et Desset, 1966) all of them are restricted to some eastern and australian Muridae. 
Deux seules espèces sont, à notre connaissance, actuellement rangées dans le genre Nippostrongylus Lane, 1923. Ce sont: N. brasiliensis (Travassos, 1914), parasite cosmopolite des rats domestiques et $N$. rauschi Chabaud et Desset, 1966, parasite de Dermoptères aux Philippines.

L'envoi d'Héligmosomes parasites de Muridés, originaires des Indes et de Chine (1), nous a amenée à reconsidérer la définition du genre Nippostrongylus en tenant compte du synlophe qui n'avait pas encore été utilisé en systématique.

La connaissance de ce synlophe, jointe aux caractères de la bourse caudale nous a permis de regrouper, dans le genre Nippostrongylus, quelques espèces décrites dans différents genres, toutes parasites de Muridés.

Le genre Nippostrongylus apparaît donc essentiellement inféodé à certains Muridés orientaux et australiens. Seul Nippostrongylus brasiliensis (Travassos, 1914) a une répartition mondiale, et l'extension de ce parasite s'est effectuée très récemment (cf. Chabaud et Desset, 1966).

\section{I. - Etude du synlophe de Nippostrongylus brasiliensis (Travassos, 1914)}

Le matériel utilisé pour cette étude provient de spécimens parasites de Rattus rattus L. originaires de Calcutta, Indes (tube $478 \mathrm{M}$ ).

Le corps est enroulé en spirale le long de la ligne ventrale. Cet enroulement n'est jamais serré et comporte 2 à 3 tours de spire. Souvent la partie postérieure est complètement déroulée.

Chez les deux sexes, le corps est parcouru longitudinalement par 14 arêtes cuticulaires qui, dans sa partie moyenne, se répartissent comme suit : 1 grande arête gauche, 1 grande arête droite, 7 arêtes ventrales, 5 arêtes dorsales (fig. 1 F).

Les arêtes naissent pour la plupart sur le bord postérieur de la vésicule céphalique (fig. $1 \mathrm{~A}-\mathrm{B}$ ). Chez le $\hat{\delta}$, les arêtes dorsales disparaissent les premières à environ $200 \mu$ en avant de la bourse caudale, puis les arêtes ventrales à $100 \mu$ (fig. 1 C). Chez la $\uparrow$, les arêtes s'étendent jusqu'au niveau de la vulve (fig. $1 \mathrm{E}$ ).

En coupe transversale au milieu du corps, la pointe des arêtes est dirigée de la ligne ventrale-droite vers la ligne dorsale-gauche pour les deux faces (fig. $1 \mathrm{~F}$ ).

Chez les deux sexes, dans la partie postérieure du corps, les arêtes diminuent de taille et sont orientées normalement à la paroi (fig. $1 \mathrm{E}$ ).

Le gradient de taille des arêtes est latéro-médian pour les quatre faces.

II. - Redéfinition du genre Nippostrongylus Lane, 1923

SynONYME : Austroheligmonema Mawson, 1961.

Heligmosomatidae avec des arêtes cuticulaires orientées de la ligne ventraledroite vers la ligne dorsale-gauche pour les deux faces. Le gradient de taille des arêtes est latéro-médian au moins pour la face dorsale et la face ventrale-gauche. Le étudié ici.

(1) Nous remercions vivement $\mathrm{M}^{\mathrm{me}}$ Erhardova, MM. Schad et Inglis pour l'envoi du matériel 

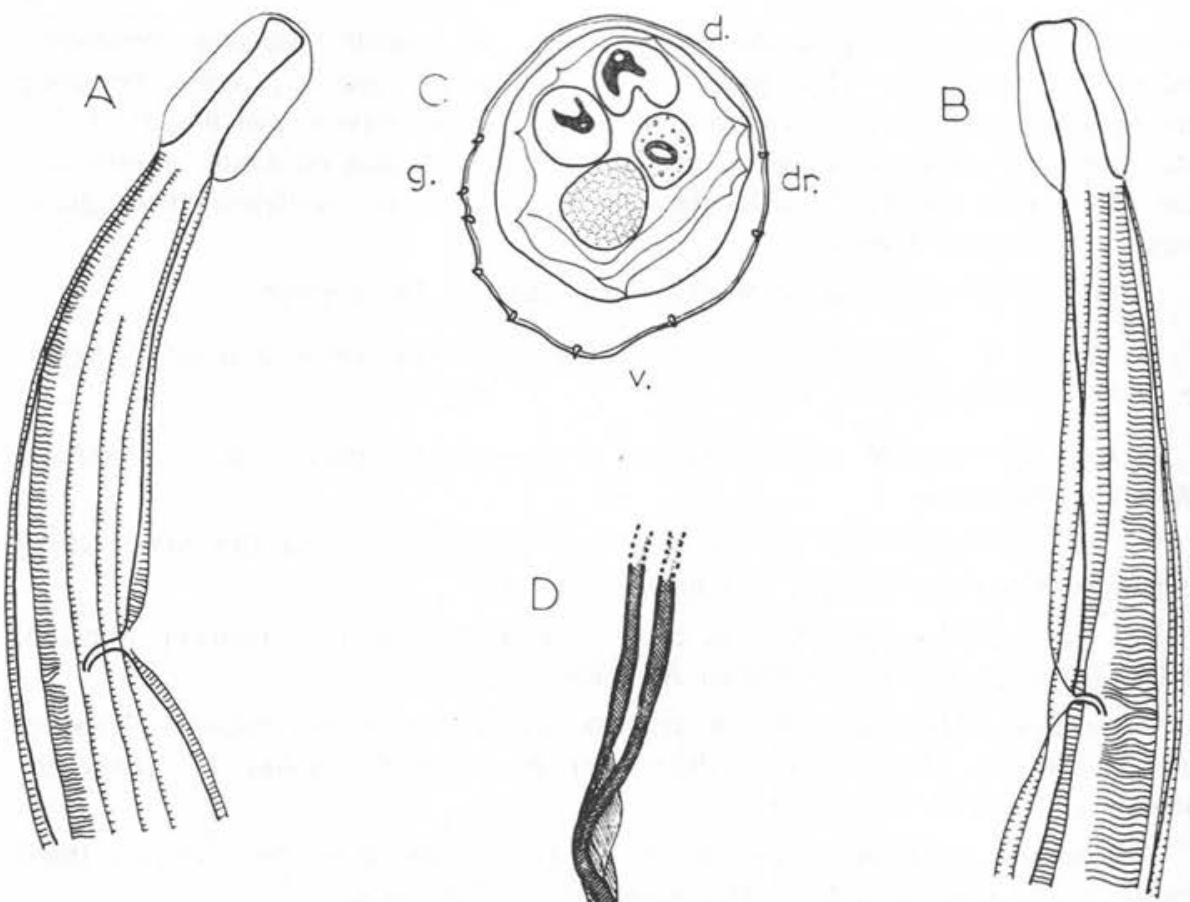

$100 \mu$
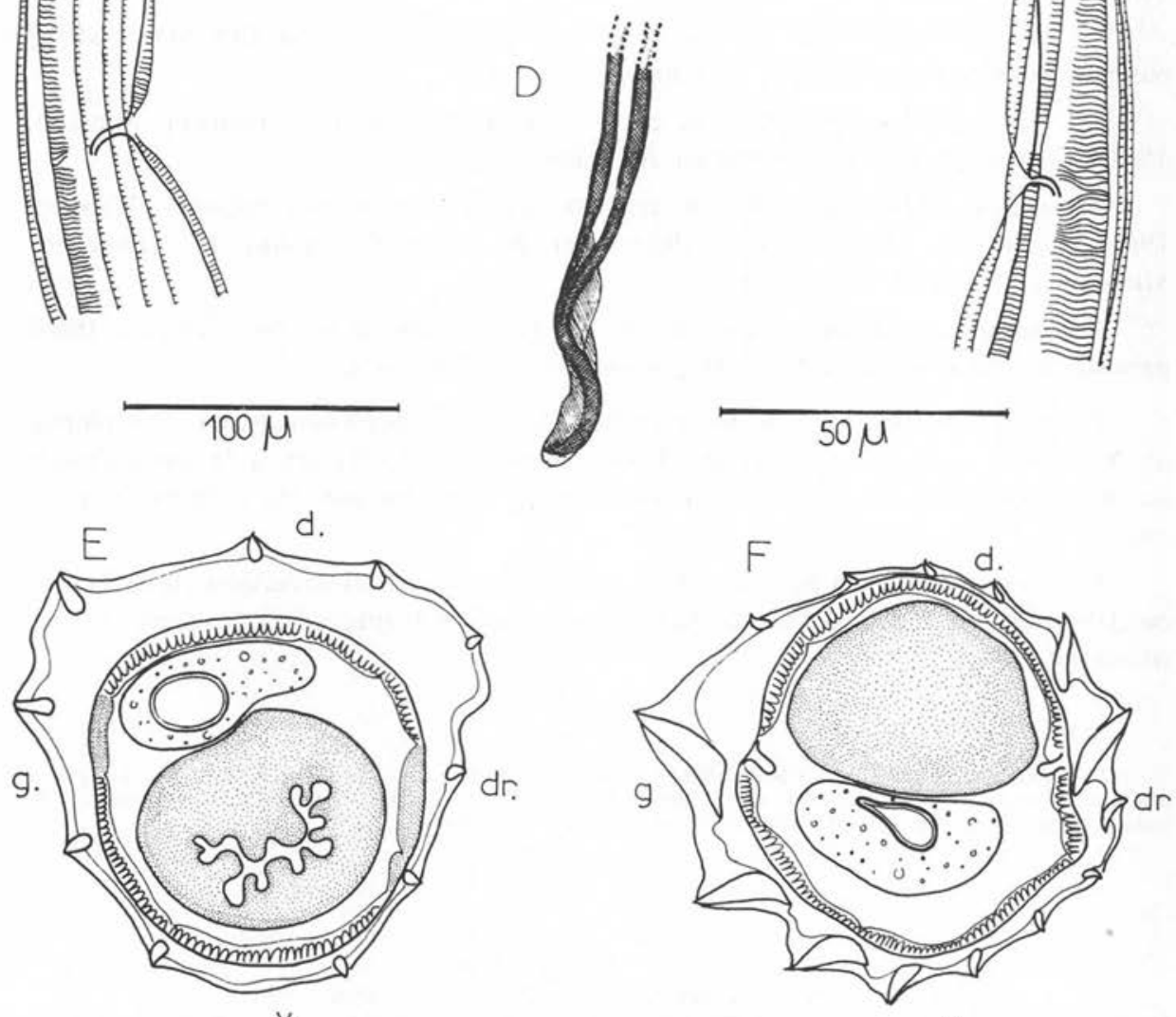

V.

Fig. 1. - Nippostrongylus brasiliensis (Travassos, 1914)

A : $\delta$, naissance des arêtes cuticulaires, extrémité antérieure, vue latérale droite. - B : $i d$, vue

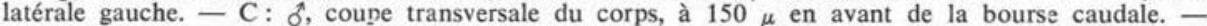
D : ठ, pointe d'un spicule. - E : $\mathcal{f}$, coupe transversale du corps au niveau du sphincter. $-\mathrm{F}: \delta$, coupe transversale au milieu du corps A, B : éch. $100 \mu$ C, D, E, F : éch. $50 \mu$ 
nombre de ces arêtes est généralement de 14. Bourse caudale fortement asymétrique avec un lobe droit plus développé. Côte postéro-latérale gauche, épaisse, beaucoup plus développée que la côte postéro-latérale droite. Côte dorsale, peu longue, divisée distalement en 4 rameaux. Côtes externo-dorsales naissant plus ou moins asymétriquement sur la côte dorsale. Femelle avec vulve s'ouvrant très postérieurement. Queue courte, généralement tronquée.

Parasites essentiellement de Muridae mais aussi de Dermoptères.

EsPÈCE-TYPE : Nippostrongylus brasiliensis (Travassos, 1914) parasite cosmopolite de rats domestiques (2).

Autres EsPèces : $N$. rauschi Chabaud et Desset, 1966, parasite de Cynocephalus volans aux Philippines.

N. rysavyi (Erhardova, 1959) n. cb. (= Oswaldonema rysavyi Erhardova, 1959) parasite de Rattus alexandrinus en Chine.

N. typicus (Mawson, 1961) n. cb. (= Austroheligmonema typicum Mawson, 1961), parasite de Rattus assimilis en Australie.

N. magnus (Mawson, 1961) n. cb. (= Austroheligmonema magnum, Mawson, 1961), parasite de Rattus assimilis (hôte-type), $R$. rattus, $R$. conatus, $R$. norvegicus, Melomys cervinipes en Australie.

N. djumachani (Tenora, 1969) n. cb. (= Longistriata djumachani Tenora, 1969) parasite de Nesokia indica et de Mus musculus en Afghanistan.

Parmi ces espèces outre $N$. brasiliensis et $N$. rauschi nous connaissons le synlophe de $N$. typicus et de $N$. magnus (of. Durette-Desset, 1970). Grâce à la complaisance de $\mathrm{M}^{\mathrm{me}}$ Erhardova, qui a envoyé le matériel, nous décrivons plus bas celui de $N$. rysavyi.

Nous ne connaissons pas celui de $N$. djumachani mais les caractères de la bourse caudale, nous paraissent suffisants pour fouvoir ranger l'espèce dans le genre Nippostrongylus.

(2) Dans une collection, aimablement communiquée par le Docteur Schad, et originaire des Indes, nous trouvons l'espèce chez Rattus rattus, $R$. norvegicus, Bandicota bengalensis et $B$. indica; cependant, elle n'est réellement abondante que chez Rattus rattus.

FIG. 2. - Nippostrongylus rysavyi (Erhardova, 1959) n. cb.

1) $4^{e}$ stade larvaire $\delta_{\text {. }}-\mathrm{A}$ : extrémité antérizure, vue latérale droite. - B : vésicule céphalique du $\delta^{*}$, dans la cuticule du $4^{e}$ stade, vue latérale droite. - C : coupe transversale au milieu du corps. - D: bourse caudale, vue latérale droite. - E: id, vue latérale gauche

2) Femelle. - F : extrémité postérieure, vue latérale gauche. $-G$ : coupe transversale au milieu du corps. - H: extrémité antérieure, vue latérale gauche. - I: dótail dəs arêtes cuticulaires au niveau du pore excréteur et de la deiride gauche

A, F, H: éch. $100 \mu$. B, C, D, E, G, I : éch. $50 \mu$ 

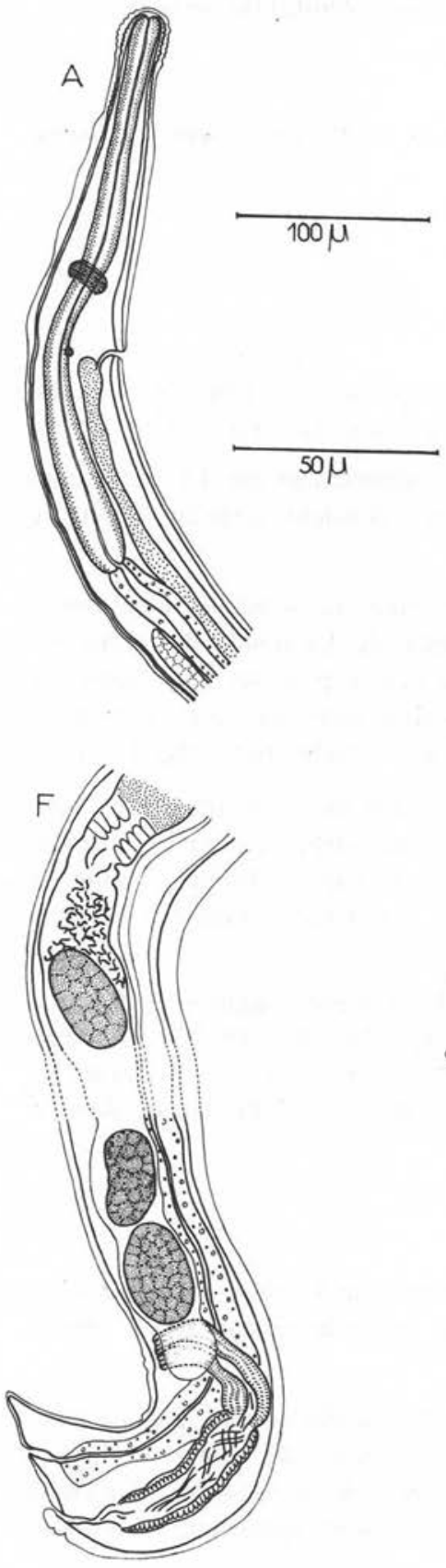
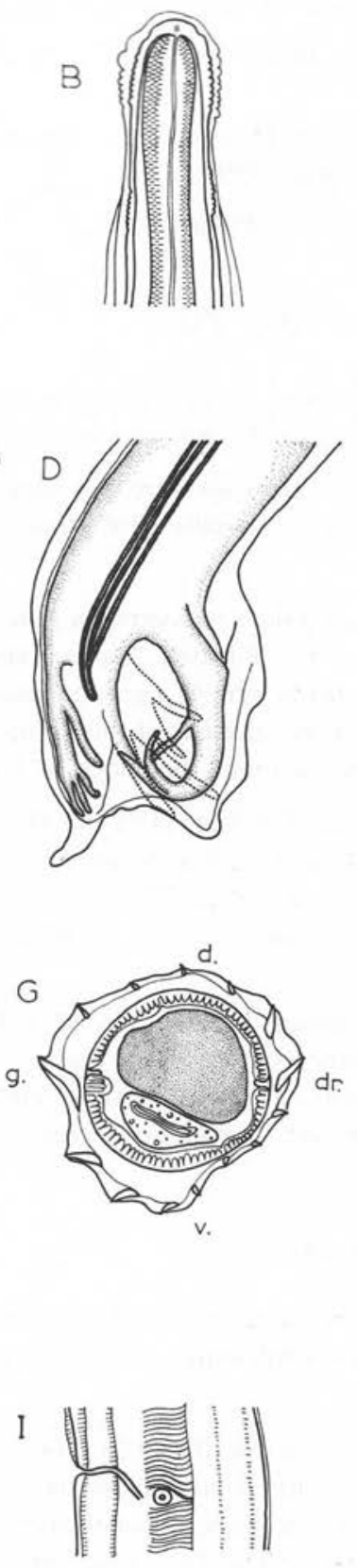
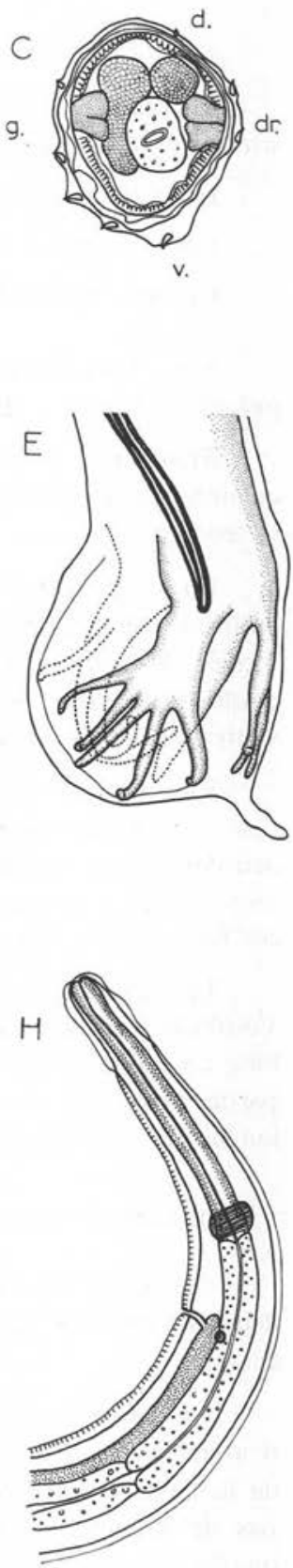


\section{III. - Compléments à la morphologie de Nippostrongylus rysavyi (Erhardova, 1959), n. cb.}

MAtÉRIEL : 3 으, un $4^{e}$ stade larvaire $\hat{o}$ (matériel type), coparasites d'Orientostrongylus chinensis (Erhardova, 1959).

Hôte : Cricetulus barabensis (Pallas).

LOCALISATION : Intestin.

Origine géographique: Pékin, Chine.

Nous complétons simplement l'étude détaillée d'Erhardova en donnant quelques précisions sur la $\&$ et en étudiant le synlophe et le quatrième stade larvaire $\hat{\delta}$.

Synlophe: Chez la + , le corps est parcouru longitudinalement par 14 arêtes cuticulaires qui débutent derrière la vésicule céphalique et s'étendent jusqu'au niveau de la trompe.

En coupe transversale au milieu du corps, la répartition des arêtes est la suivante : 2 fortes arêtes latérales, 5 arêtes dorsales, 7 arêtes ventrales. La pointe des arêtes est dirigée de la ligne ventrale-droite vers la ligne dorsale-gauche pour les deux faces. Le gradient de taille des arêtes est nettement latéro-médian pour les faces dorsale et ventrale-gauche. Ce gradient est moins net pour la face ventrale-droite (fig. $2 \mathrm{G}$ ).

Femelle : Corps long de $1,8 \mathrm{~mm}$, large de $60 \mu$ dans sa partie moyenne. Vésicule céphalique haute de $50 \mu$ sur $25 \mu$ de large. Anneau nerveux, pore excréteur et deirides situées respectivement à $135 \mu, 175 \mu$ et $180 \mu$ de l'apex. Esophage long de $360 \mu(200 \mu$ œsophage musculaire ; $160 \mu$ œsophage glandulaire). Glandes excrétrices bien visibles (fig. $2 \mathrm{H}$ ).

La vulve s'ouvre à l'extérieur de la spire à $67 \mu$ de la queue. Vagin long de $50 \mu$. Vestibule, sphincter et trompe longs respectivement de $62 \mu, 20 \mu$ et $70 \mu$. L'utérus long de $330 \mu$ contenait 6 œufs au stade morula, hauts de $50 \mu$ sur $30 \mu$ de large. La partie proximale de l'utérus est emplie de spermatozoïdes (fig. 2 F). Queue épaisse, longue de $32 \mu$ (fig. 2 F).

\section{QUATRIÈME STADE LARVAIRE $\hat{o}$}

SyNLOPHE : Le corps est parcouru longitudinalement par 8 arêtes cuticulaires qui débutent derrière la vésicule céphalique et s'étendent jusqu'au niveau de la bourse caudale.

En coupe transversale au milieu du corps, ces arêtes se répartissent comme suit : 4 arêtes ventrales, 1 arête droite, 3 arêtes dorsales. La pointe des arêtes est dirigée de la ligne ventrale-droite vers la ligne dorsale-gauche pour les deux faces. Il n'existe pas de gradient de taille des arêtes. Les arêtes ventrales sont légèrement plus développées que les arêtes dorsales (fig. $2 \mathrm{C}$ ). 
Mensurations: Longueur 1,7 mm; largeur dans la partie moyenne du corps: $50 \mu$. Vésicule céphalique : $50 \mu$ de haut sur $20 \mu$ de large. Anneau nerveux, pore excréteur, deirides situés respectivement à $130 \mu, 175 \mu$ et $175 \mu$ de l'apex. CEsophage : $280 \mu$. Spicules : $330 \mu$ (fig. 2 D-E).

Nous avons identifié le quatrième stade larvaire $\hat{o}$ comme appartenant au genre Nippostrongylus et non à Orientostrongylus chinensis (Erhardova, 1959)) étant donné la présence d'un grand lobe gauche visible même à l'intérieur de la cuticule du quatrième stade (fig. 2 , D) et de la longueur des spicules $(330 \mu)$ alors que chez le $\hat{o}$ adulte de $O$. chinensis, ils sont au maximum de $95 \mu$.

\section{Bibliographie}

Chabaud (A.-G.) et Desset (M.-C.), 1966. - Nippostrongylus rauschi n. sp. Nématode parasite de Dermoptères et considérations sur $N$. brasiliensis, parasite cosmopolite des Rats domestiques. Ann. Par. Hum. Comp., 41 (3), 243-249, fig. 1-3.

DuretTe-Desset (M.-C.), 1970. - Les systèmes d'arêtes cuticulaires chez les Nématodes Héligmosomes, parasites de Muridés australiens. Ann. Par. Hum. Comp., 44 (6), 733-747, fig. 1-8.

ERHardova (B.), 1959. - Oswaldonema rysavyi n. sp. und Vianella chinensis n. sp. (Nematoda: Heligmosomatidae) bei chinesischen nagern. Ceskoslovenska parasitologie, 6 (1), 93-96, fig. 1-3.

LANE (C.), 1923. - Some strongylata. Parasitology, 15, 348-364, fig. 1-80.

Mawson (P.-M.), 1961. - Trichostrongyles from Rodents in Queensland, with comments on the genus Longistriata (Nematoda : Heligmosomatidae). Austr. J. Zool., 9 (5), 791 826, fig. 1-64.

Tenora (F.), 1969. - Parasitic Nematodes of certain Rodents from Afghanistan. Vèstnik. Cs. spol. Zool. (Acta Soc. Zool. Bohemoslov), 33 (2), 174-192, fig. 1-5.

Travassos (L.), 1914. - Trichostrongylideos brazileiros (III. nota previa). Brazil. Med., 28 (34), 325-327. 